\title{
High MACC1 expression in combination with mutated KRAS G13 indicates poor survival of colorectal cancer patients
}

Katharina $\| \mathrm{m}^{1 \dagger}{ }^{\dagger}$, Wolfgang Kemmner ${ }^{1 \dagger}$, Marc Osterland ${ }^{1}$, Susen Burock ${ }^{2}$, Gudrun Koch? ${ }^{1}$, Pia Herrmann ${ }^{1}$, Peter M Schlag ${ }^{2}$ and Ulrike Stein ${ }^{1,3^{*}}$

\begin{abstract}
Background: The metastasis-associated in colon cancer 1 (MACC1) gene has been identified as prognostic biomarker for colorectal cancer (CRC). Here, we aimed at the refinement of risk assessment by separate and combined survival analyses of MACC1 expression with any of the markers KRAS mutated in codon 12 (KRAS G12) or codon 13 (KRAS G13), BRAF V600 mutation and MSI status in a retrospective study of 99 CRC patients with tumors UICC staged II II and III.

Findings: We showed that only high MACC1 expression (HR: 6.09, 95\% Cl: 2.50-14.85, P < 0.001) and KRAS G13 mutation (HR: 5.19, 95\% Cl: 1.06-25.45, $\mathrm{P}=0.042$ ) were independent prognostic markers for shorter metastasis-free survival (MFS). Accordingly, Cox regression analysis revealed that patients with high MACC1 expression and KRAS G13 mutation exhibited the worst prognosis (HR: 14.48, 95\% Cl: 3.37-62.18, P < 0.001). Patients were classified based on their molecular characteristics into four clusters with significant differences in MFS ( $P=0.003)$ by using the SPSS 2-step cluster function and Kaplan-Meier survival analysis.

Conclusion: According to our results, patients with high MACC1 expression and mutated KRAS G13 exhibited the highest risk for metachronous metastases formation. Moreover, we demonstrated that the "Traditional pathway" with an intermediate risk for metastasis formation can be further subdivided by assessing MACC1 expression into a low and high risk group with regard to MFS prognosis. This is the first report showing that identification of CRC patients at high risk for metastasis is possible by assessing MACC1 expression in combination with KRAS G13 mutation.
\end{abstract}

Keywords: KRAS mutations, MACC1, Prognostic marker, Colorectal cancer, Metastasis-free survival

\section{Findings}

\section{Background}

Colorectal cancer (CRC) is the third most common cancer worldwide [1]. According to the state of the art for classification, colorectal carcinoma can be divided into three major pathways based on the underlying molecular alterations $[2,3]$. The majority of CRC belong to the "Traditional pathway" which reflects the adenomacarcinoma sequence model $[4,5]$. Two additional

\footnotetext{
* Correspondence: ustein@mdc-berlin.de

${ }^{\dagger}$ Equal contributors

${ }^{1}$ Experimental and Clinical Research Center, Charité University Medicine

Berlin and Max-Delbrück-Center for Molecular Medicine, Robert-Rössle-Str.10, 13125 Berlin, Germany

${ }^{3}$ German Cancer Consortium, Heidelberg, Germany

Full list of author information is available at the end of the article
}

pathways where recently described, the "Serrated" and "Alternate pathway" [2-4], which are characterized by differences in the microsatellite instability (MSI) status and mutations in the KRAS or BRAF oncogenes. In advanced stages metastatic spread shortens the 5-year survival rate from $90 \%$ to $10 \%[6,7]$. Risk estimation by using genetic factors for early identification of high risk patients is still limited [8].

One promising candidate is the metastasis-associated in colon cancer 1 (MACC1) gene. MACC1 is an important prognostic marker for metastases formation in CRC $[9,10]$.

Other promising molecular markers are oncogenic KRAS mutations, which are detectable in $30-50 \%$ of CRC tumors [11]. The most frequent point mutations in codon 12 (KRAS G12) and 13 (KRAS G13) of exon 2 
result in constitutive activation of KRAS and downstream pathways [12]. Although the role of KRAS mutations as predictive biomarkers for anti-EGFR-targeted therapy is well characterized, there is a need to clarify their relevance as prognostic biomarkers for recurrence of metastatic CRC [13].

In this retrospective study, we attempted to improve the prognostic value of MACC1 for CRC metastasis using separate and combined survival analyses of MACC1 expression levels and KRAS G12 or G13 mutations in tumors of CRC patients. In addition, we investigated the prognostic significance of BRAF V600 mutation and MSI status, two of the most promising prognostic and predictive markers in the landscape of molecular biomarkers.

\section{Associations of MACC1 expression with the KRAS G12, KRAS G13 and BRAF V600 mutations as well as MSI status} In total, 99 patients with patho-histologically confirmed primary colorectal adenocarcinomas with Union for International Cancer Control (UICC) stages I, II and III were included in this study. This patient study protocol was approved by the Institutional Review Board (IRB) of the Charité Universitätsmedizin Berlin, complied with the Helsinki Declaration. The median metastasis-free survival (MFS) time and overall survival (OS) time of the patients were 56.0 and 79.4 months, respectively. This tumor marker study based on hypothesis-driven approaches was designed in compliance with the REMARK guidelines [14]. MACC1 mRNA expression level of the tumors were quantified using quantitative reverse transcriptase PCR [9]. All patient and tumor characteristics are shown in detail in the Additional file 1: Table S1-S4. For KRAS and BRAF mutation analyses as well as MSI status determination DNA sequencing methods were used $[15,16]$. All used material and methods are described in detail in Additional file 2. The molecular characteristics in this cohort (Additional file 1: Table S2) demonstrate the general distribution within colorectal tumors and mutual exclusivity of the oncogenes KRAS and BRAF $[7,15]$.

MACC1 expression was significantly higher in primary tumors of patients who developed metachronous metastases during the follow-up period $(\mathrm{P}=0.009$; Figure $1 \mathrm{~A})$, which confirmed previously described coherences $[9,10]$. We showed for the first time a correlation of high MACC1 expression with tumors harboring KRAS mutations (G12 or G13) $(\mathrm{P}=0.049$; Figure 1B) [7]. Separate analyses of primary tumors harboring the KRAS G13 mutation exhibited a significantly higher MACC1 expression $(P=0.010$; Figure $1 D)$, but not for KRAS G12 mutated tumors $(\mathrm{P}=0.156$; Figure $1 \mathrm{C})$. High MACC1 expression also correlated with BRAF wild type (wt) $(\mathrm{P}=0.027$; Figure $1 \mathrm{E})$ and microsatellite stable (MSS)/MSI-Low (MSI-L) $(\mathrm{P}=0.009$; Figure $1 \mathrm{~F})$ tumors.

\section{Association of MACC1 expression, KRAS G12 or G13 mutation, BRAF mutation and MSI status with MFS}

We performed Kaplan-Meier survival analyses (Figure 2) and univariate Cox regression to estimate hazard ratios (HR) and 95\% confidence intervals (CI) for the association of the tumor characteristics with MFS (Additional file 1: Table S5). Interestingly, univariate Cox regression and Log Rank test revealed that in the set of analyzed markers, only high MACC1 expression levels and KRAS G13 mutation emerged as prognostic factors for MFS. Patients with high MACC1 expression levels (Figure 2A, Log Rank $\mathrm{P}<0.001$; Cox regression: low vs. high, HR: 5.02, 95\% CI: $2.19-11.53, \mathrm{P}<0.001)$ or KRAS G13 mutation (Figure 2D, $\log$ Rank $\mathrm{P}=0.022$; Cox regression: wt vs. KRAS G13, HR: $4.25,95 \%$ CI: $1.21-14.93, \mathrm{P}=0.024$ ) in their primary tumors exhibited a significantly poorer prognosis. Although the role of KRAS G13 mutation as a predictive biomarker for anti-EGFR-targeted therapy [18] is well characterized, the prognostic value of KRAS G13 mutation in patients with CRC is controversial [19]. Various reports either encourage or dismiss KRAS mutations as prognostic biomarker for disease-specific survival rates or to predict liver or lung metastases [7,19-24]. Data of the Kirsten Ras in Colorectal Cancer Collaborative Group Studies (RASCAL I) suggested that the risk of recurrence and death is increased by the presence of KRAS mutations [11], and the updated RASCAL II study confined the correlation of KRAS mutation and survival prognosis to the G12V mutation [25]. According to our results, comparison of mutated KRAS tumors with KRAS wt tumors had no significant impact on MFS (Figure 2B, Log Rank P = 0.499; Cox regression: wt vs. KRAS G12 or G13, HR: 1.40, 95\% CI: 0.63-3.09, P = 0.409). Studies comparing the effects of KRAS G12 with KRAS G13 mutations are rare [19,24]. Therefore separate analyses of KRAS G12 and KRAS G13 mutations in the tumors were included and demonstrated that especially the KRAS G13, but not KRAS G12 (Figure 2C, Log Rank P = 0.654; Cox regression: wt vs. KRAS G12, HR: 1.29, 95\% CI: 0.55-3.03, $\mathrm{P}=0.556$ ), mutations were prognostic for reduced MFS. Separate analyses of the most prominent G12 mutations (G12V, G12D and G12C) revealed no significant impact of these mutations on MFS prognosis (Log Rank $\mathrm{P}=0.826$, $\mathrm{P}=0.896$ and $\mathrm{P}=0.766$; respectively). Furthermore, BRAF V600 mutation (Figure 2E, Log Rank $\mathrm{P}=0.656$; Cox regression: wt vs. BRAF V600, HR: 0.75, 95\% CI: 0.18-3.17, $\mathrm{P}=0.694$ ) or MSS/MSI-L (Figure $2 \mathrm{~F}$, Log Rank $\mathrm{P}=0.085$; Cox regression: MSS/MSI-L vs. MSI-H, HR: 0.04, 95\% CI: $0.00-12.05, \mathrm{P}=0.272$ ) were also not associated with worse prognosis. 


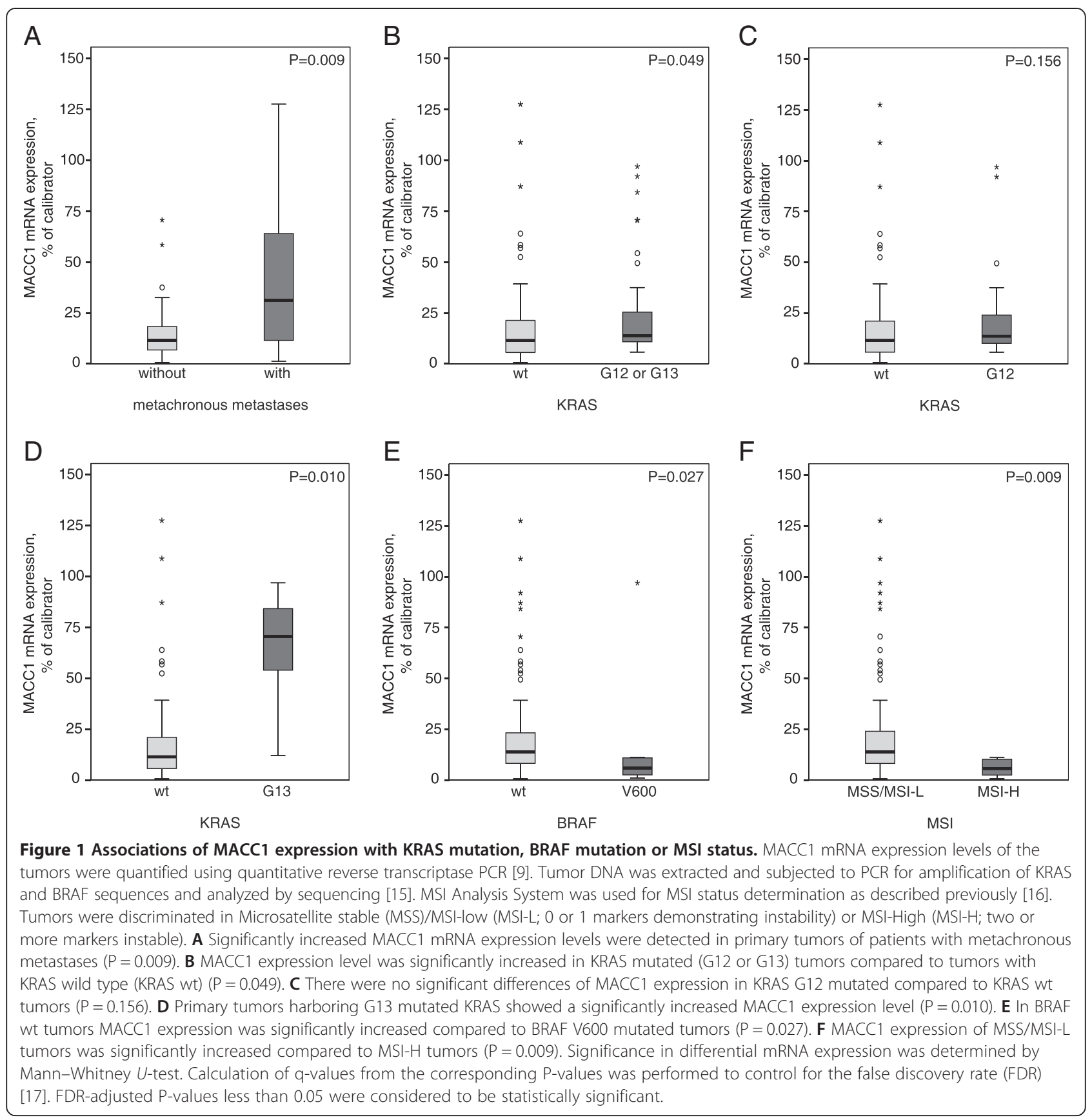

Combinatorial survival analyses of MACC1 expression and KRAS G12 or G13 mutation with tumor characteristics

Next, we analyzed the effects of clinicopathological parameters like age, gender, UICC stage, grading and postoperative treatment of the tumors concerning MFS in combination with MACC1 or KRAS mutations using multivariate Cox regression (Table 1). Only high MACC1 expression (HR: 6.09, 95\% CI: 2.50-14.85, $\mathrm{P}<0.001$ ) and KRAS G13 mutation (HR: 5.19, 95\% CI: $1.06-25.45, \mathrm{P}=0.042$ ) turned out to be independent prognostic markers for shorter MFS in the set of analyzed biomarkers. To our knowledge, this is the first study showing a prognostic relevance of KRAS G13 for MFS in CRC patients and demonstrating KRAS G13 mutation as independent risk factor of age, gender, UICC stage, grading and postoperative treatment for metachronous metastases development.

In addition, we performed multivariate Cox regression combining MACC1 expression level with the tumor characteristics KRAS G12 or G13 mutation, BRAF V600 mutation and MSI status (Additional file 1: 


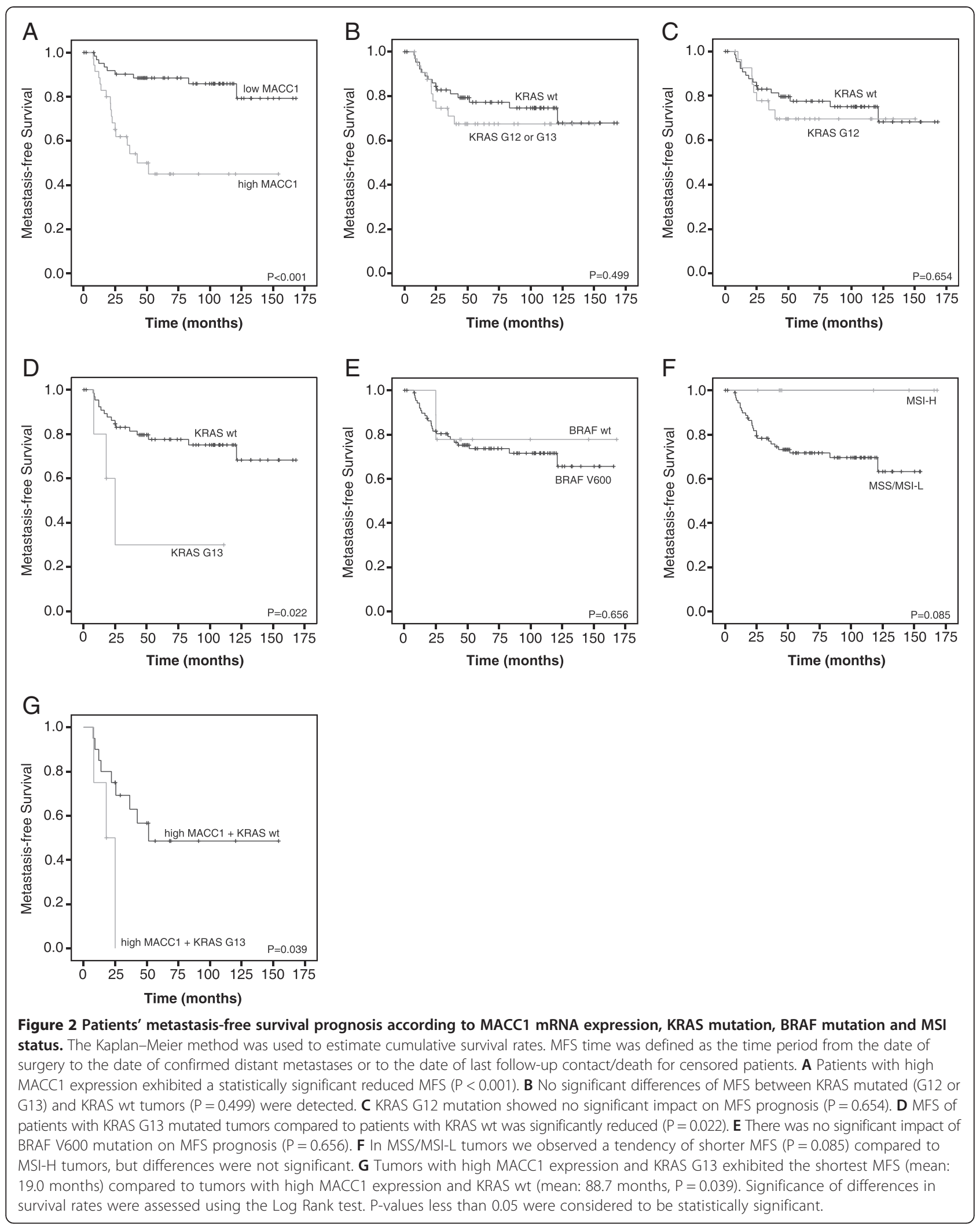


Table 1 Impact of clinicopathological parameters in combination with MACC1 and KRAS mutation status concerning MFS

\begin{tabular}{|c|c|c|c|c|c|c|c|c|}
\hline \multirow[t]{2}{*}{ Covariates } & \multicolumn{2}{|c|}{$\begin{array}{l}\text { Multivariate } \\
\text { MACC1 }\end{array}$} & \multicolumn{2}{|c|}{$\begin{array}{l}\text { Multivariate mutated } \\
\text { (G12 or G13) KRAS }\end{array}$} & \multicolumn{2}{|c|}{$\begin{array}{l}\text { Multivariate G13 } \\
\text { mutated KRAS }\end{array}$} & \multicolumn{2}{|c|}{$\begin{array}{l}\text { Multivariate G12 } \\
\text { mutated KRAS }\end{array}$} \\
\hline & P-value & $\mathrm{HR}(95 \% \mathrm{Cl})$ & P-value & HR $(95 \% \mathrm{Cl})$ & P-value & $\mathrm{HR}(95 \% \mathrm{Cl})$ & P-value & $\mathrm{HR}(95 \% \mathrm{Cl})$ \\
\hline $\begin{array}{l}\text { Molecular Markers MACC1 (low vs. high) } \\
\text { KRAS G12/G13 (wt vs. mutated) }\end{array}$ & $<0.001$ & $6.09(2.50-14.85)$ & 0.302 & $1.55(0.67-3.59)$ & 0.042 & $5.19(1.06-25.45)$ & 0.543 & $1.34(0.52-3.42)$ \\
\hline Age at diagnosis $(<60)$ vs. $(>60)$ years & 0.970 & $1.02(0.41-2.52)$ & 0.892 & $0.94(0.39-2.26)$ & 0.648 & $1.30(0.43-3.93)$ & 0.940 & $1.04(0.37-2.90)$ \\
\hline By gender Male vs. Female & 0.890 & $0.94(0.41-2.17)$ & 0.969 & $1.02(0.44-2.34)$ & 0.494 & $1.42(0.52-3.91)$ & 0.963 & $0.98(0.41-2.35$ \\
\hline UICC stage (I) vs. (II + III) & 0.473 & $1.65(0.42-6.53)$ & 0.191 & $2.42(0.64-9.09)$ & 0.356 & $2.16(0.42-11.10)$ & 0.115 & $3.59(0.73-17.54)$ \\
\hline 3y grading $(\mathrm{G} 1+2)$ vs. $(\mathrm{G} 3+4)$ & 0.416 & $0.64(0.22-1.88)$ & 0.257 & $0.53(0.18-1.59)$ & 0.305 & $0.45(0.10-2.06)$ & 0.094 & $0.28(0.06-1.24)$ \\
\hline $\begin{array}{l}\text { By postoperative treatment Untreated } \\
\text { vs. treated }\end{array}$ & 0.064 & $2.37(0.95-5.93)$ & 0.447 & $1.40(0.59-3.29)$ & 0.723 & $1.22(0.41-3.57)$ & 0.483 & $1.39(0.55-3.49)$ \\
\hline
\end{tabular}

The P-values, hazard ratios (HR) and 95\% confidence intervals (Cl) of different parameters concerning MFS were calculated using Cox regression analyses. The analysis was performed separately for each factor with the parameters listed in the table being introduced as covariates, respectively.

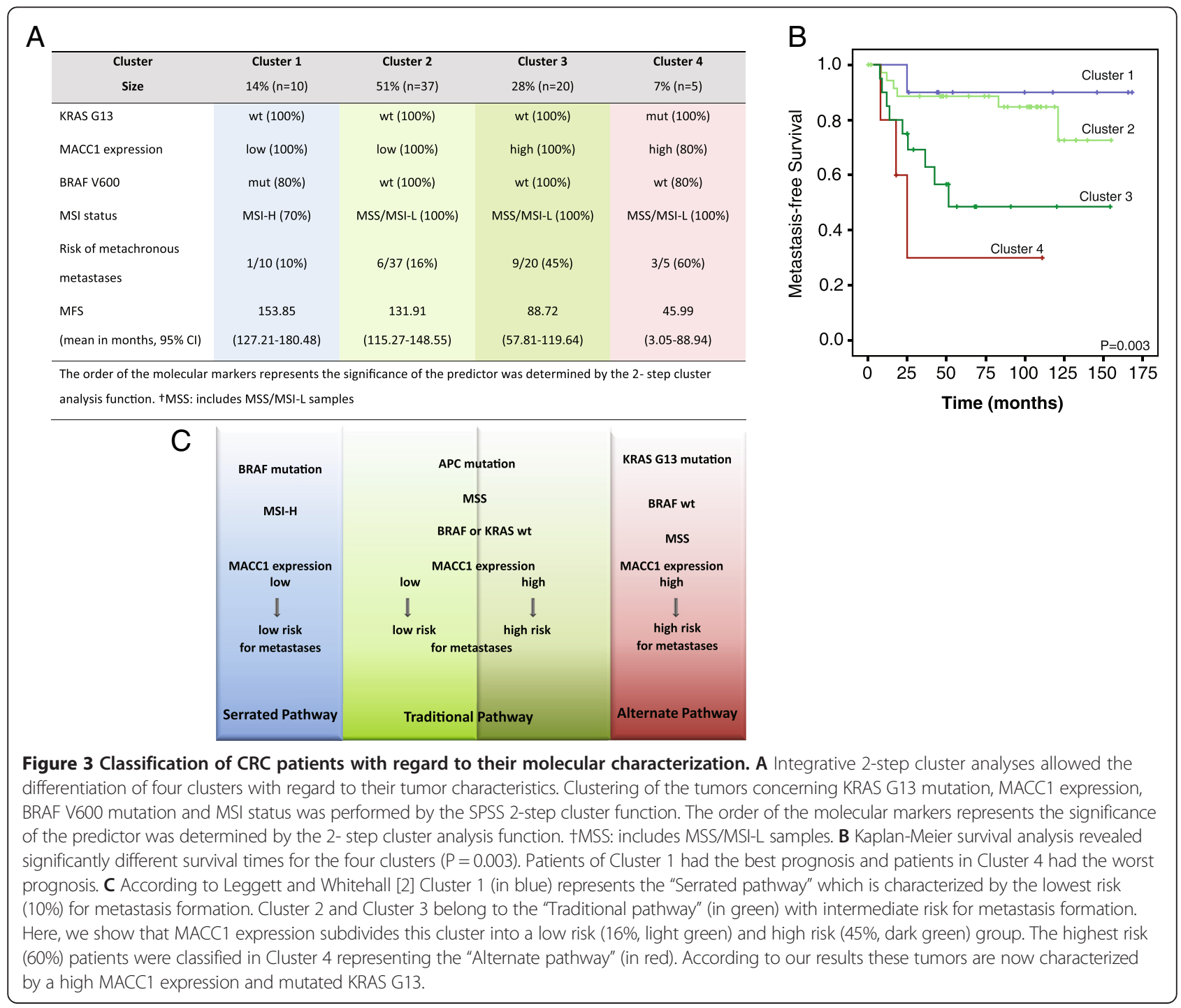


Table S5). This analysis revealed that MACC1 was statistically significant independent for MFS prognosis of these molecular markers (HR: 4.20, 95\% CI: 1.80-9.81, $\mathrm{P}=0.001)$. Therefore, we conducted combinatorial survival prognosis of the independent prognostic markers MACC1 expression and KRAS G13 mutation. Interestingly, patients with the worst prognosis were characterized by high MACC1 expression and KRAS G13 mutation (HR: 14.48, 95\% CI: 3.37-62.18, $\mathrm{P}<0.001)$. These patients had the shortest MFS time (Figure 2G, Log Rank: $\mathrm{P}=0.039$ mean: 18.99 months, 95\% CI: 10.64-27.34) compared to patients with high MACC1 expression and KRAS wt tumors (Additional file 1: Table S6). The identification of CRC patients at high risk for metastasis formation is possible by assessing MACC1 expression in combination with KRAS G13 mutations.

\section{Cluster classification}

Using integrative 2-step cluster analysis based on the presence of the proven markers in our cohort four clusters of patients with a different risk for metastasis formation could be distinguished and coincide well with the presumed pathways (Figure 3). According to CRC classification by Leggett and Whitehall [2], Cluster 1 represents the "Serrated pathway" with MSI-H, BRAF mutation and low MACC1 expression. These patients showed the lowest risk for metachronous metastases formation. In our study Cluster 2 and 3 represents the "Traditional pathway", where the majority of CRC cases belong to. This pathway is characterized by MSS and KRAS or BRAF mutation. Cluster 2 with a low risk for metastasis formation was further characterized by low MACC1 expression. In contrast, Cluster 3 with increased risk exhibited high MACC1 expression levels. Thus, MACC1 expression allows a further subdivision of the "Traditional pathway" into a low and high risk group of patients concerning metastasis formation. Patients in Cluster 4 had the highest risk for metachronous metastases development. These tumors belonging to the "Alternate pathway" were defined by MSI-L/MSS, KRAS G13 mutation and can be further characterized by high MACC1 expression.

\section{Conclusion}

This exploratory study has limitations especially due to the limited number of patient samples. But this cohort has also several strengths: The marker analyses were performed with microdissected cryosections verified by an experienced pathologist. Furthermore the studied cohort is very well characterized and only samples from preoperatively untreated patients with very long follow-up data and complete clinicopathological information were used.

In conclusion, our results underline the high relevance of MACC1 and KRAS G13 mutation for CRC metastasis prognosis. This is the first study showing a prognostic relevance of KRAS G13 for MFS in CRC patients. Furthermore, high MACC1 expression and KRAS G13 mutation are independent prognostic markers for metachronous metastases development. Accordingly, combinatorial survival prognosis revealed that patients with high MACC1 expression and KRAS G13 mutation exhibited the worst prognosis.

Interestingly, the conventional CRC classification can be extended and refined by inclusion of MACC1 expression and mutated KRAS G13 as independent markers. Therefore, this refinement allows improved individualized prognosis and intervention strategies for the majority of CRC patients in clinical routine.

\section{Consent}

Written informed consent was obtained from the patient for the publication of this report and any accompanying images.

\section{Additional files}

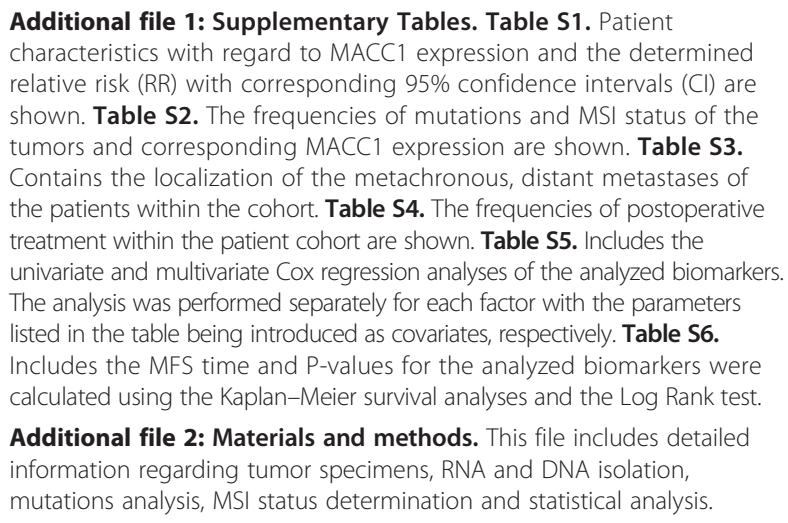
relative risk (RR) with corresponding 95\% confidence intervals $(\mathrm{Cl})$ are shown. Table S2. The frequencies of mutations and MSI status of the tumors and corresponding MACC1 expression are shown. Table S3. Contains the localization of the metachronous, distant metastases of the patients within the cohort. Table S4. The frequencies of postoperative treatment within the patient cohort are shown. Table S5. Includes the univariate and multivariate Cox regression analyses of the analyzed biomarkers. The analysis was performed separately for each factor with the parameters listed in the table being introduced as covariates, respectively. Table S6. Includes the MFS time and P-values for the analyzed biomarkers were calculated using the Kaplan-Meier survival analyses and the Log Rank test.

Additional file 2: Materials and methods. This file includes detailed information regarding tumor specimens, RNA and DNA isolation, mutations analysis, MSI status determination and statistical analysis.

\section{Abbreviations}

BRAF: v-Raf murine sarcoma viral oncogene homolog B; Cl: Confidence interval; CRC: Colorectal cancer; EGFR: Epidermal growth factor receptor; HR: Hazard ratio; FDR: False discovery rate; KRAS: Kirsten rat sarcoma viral oncogene homolog; MACC1: Metastasis-associated in colon cancer 1; MFS: Metastasis-free survival; MSI (-L/H): Microsatellite instability (-low/high); MSS: Microsatellite stable; RASCAL: Kirsten Ras in Colorectal Cancer Collaborative Group; RR: Relative risk; UICC: Union for International Cancer Control; wt: Wild type.

\section{Competing interests}

The authors certify that they have no affiliations with or involvement in any organization or entity with any financial interest or non-financial interest in the subject matter or materials discussed in this manuscript.

\section{Authors' contributions}

$\mathrm{Kl}$ and WK contributed equally as first authors. They performed the analysis, interpreted the results and wrote the manuscript. MO and SB assisted with the statistical analysis and provided critical analysis of the manuscript. GK 
and PH assisted with performance of mutation and expression analyses. US and PMS designed the study, provided clinical advice and revised the manuscript for important intellectual content. All authors critically analyzed, read and approved the final manuscript.

\section{Acknowledgments}

This work was supported by the German Cancer Consortium (DKTK) (to US). We would like to thank Susann Förster and Christoph Treese for sharing their experience in biostatistics.

\section{Author details}

'Experimental and Clinical Research Center, Charité University Medicine Berlin and Max-Delbrück-Center for Molecular Medicine, Robert-Rössle-Str.10, 13125 Berlin, Germany. ${ }^{2}$ Charité Comprehensive Cancer Center, Berlin, Germany. ${ }^{3}$ German Cancer Consortium, Heidelberg, Germany.

Received: 12 September 2014 Accepted: 5 February 2015 Published online: 14 February 2015

\section{References}

1. Ferlay J, Soerjomataram II, Dikshit R, Eser S, Mathers C, Rebelo M, et al. Cancer incidence and mortality worldwide: sources, methods and major patterns in GLOBOCAN 2012. Int J Cancer. 2015;136:E359-86.

2. Leggett $B$, Whitehall $V$. Role of the serrated pathway in colorectal cancer pathogenesis. Gastroenterology. 2010;138:2088-100.

3. Markowitz SD, Bertagnolli MM. Molecular origins of cancer: Molecular basis of colorectal cancer. N Engl J Med. 2009;361:2449-60.

4. Worthley D-L, Whitehall V-L, Spring K-J, Leggett B-A. Colorectal carcinogenesis: road maps to cancer. World J Gastroenterol. 2007;13:3784-91.

5. Fearon ER, Vogelstein B. A genetic model for colorectal tumorigenesis. Cell. 1990;61:759-67.

6. Monzon FA, Ogino S, Hammond MEH, Halling KC, Bloom KJ, Nikiforova MN. The role of KRAS mutation testing in the management of patients with metastatic colorectal cancer. Arch Pathol Lab Med. 2009;133:1600-6.

7. Nitsche U, Rosenberg R, Balmert A, Schuster T, Slotta-Huspenina J, Herrmann $P$, et al. Integrative marker analysis allows risk assessment for metastasis in stage II colon cancer. Ann Surg. 2012:256:763-71. discussion 771.

8. Deschoolmeester V, Baay M, Specenier P, Lardon F, Vermorken JB. A review of the most promising biomarkers in colorectal cancer: one step closer to targeted therapy. Oncologist. 2010;15:699-731.

9. Stein U, Walther W, Arlt F, Schwabe H, Smith J, Fichtner I, et al. MACC1, a newly identified key regulator of HGF-MET signaling, predicts colon cancer metastasis. Nat Med. 2009;15:59-67.

10. Stein U. MACC1 - a novel target for solid cancers. Expert Opin Ther Targets. 2013;17:1039-52.

11. Andreyev HJ, Norman AR, Cunningham D, Oates JR, Clarke PA. Kirsten ras mutations in patients with colorectal cancer: the multicenter "RASCAL" study. J Natl Cancer Inst. 1998:90:675-84.

12. Markman B, Javier Ramos F, Capdevila J, Tabernero J. EGFR and KRAS in colorectal cancer. Adv Clin Chem. 2010;51:71-119.

13. Er T-K, Chen C-C, Bujanda L, Herreros-Villanueva M. Clinical relevance of KRAS mutations in codon 13: Where are we? Cancer Lett. 2014;343:1-5.

14. McShane LM, Altman DG, Sauerbrei W, Taube SE, Gion M, Clark GM. REporting recommendations for tumor MARKer prognostic studies (REMARK). Nat Clin Pract Oncol. 2005:2:416-22.

15. Rosenberg DW, Yang S, Pleau DC, Greenspan EJ, Stevens RG, Rajan TV, et al. Mutations in BRAF and KRAS differentially distinguish serrated versus non-serrated hyperplastic aberrant crypt foci in humans. Cancer Res. 2007;67:3551-4.

16. Bacher JW, Flanagan LA, Smalley RL, Nassif NA, Burgart LJ, Halberg RB, et al. Development of a fluorescent multiplex assay for detection of MSI-High tumors. Dis Markers. 2004:20:237-50.

17. Storey JD, Tibshirani R. Statistical significance for genomewide studies. Proc Natl Acad Sci U S A. 2003:100:9440-5.

18. Tejpar S, Celik I, Schlichting M, Sartorius U, Bokemeyer C, Van Cutsem E. Association of KRAS G13D tumor mutations with outcome in patients with metastatic colorectal cancer treated with first-line chemotherapy with or without cetuximab. J Clin Oncol. 2012:30:3570-7.

19. Yokota T. Are KRAS/BRAF mutations potent prognostic and/or predictive biomarkers in colorectal cancers? Anticancer Agents Med Chem. 2012;12:163-71.
20. Roth AD, Tejpar S, Delorenzi M, Yan P, Fiocca R, Klingbiel D, et al. Prognostic role of KRAS and BRAF in stage $\|$ and III resected colon cancer: results of the translational study on the PETACC-3, EORTC 40993, SAKK 60-00 trial. J Clin Oncol. 2010:28:466-74.

21. Tsunoda A, lijima T, Tsunoda Y, Nakao K, Miyaki M, Kusano M. Association of K-ras mutations with liver metastases from colorectal carcinoma. Anticancer Res. 2004;24:2471-6.

22. Kim M-J, Lee HS, Kim JH, Kim YJ, Kwon JH, Lee J-O, et al. Different metastatic pattern according to the KRAS mutational status and site-specific discordance of KRAS status in patients with colorectal cancer. BMC Cancer. 2012;12:347.

23. Mannan A, Hahn-Strömberg V. K-ras mutations are correlated to lymph node metastasis and tumor stage, but not to the growth pattern of colon carcinoma. APMIS. 2012;120:459-68.

24. Rose JS, Serna DS, Martin LK, Li X, Weatherby LM, Abdel-Misih S, et al. Influence of KRAS mutation status in metachronous and synchronous metastatic colorectal adenocarcinoma. Cancer. 2012;118:6243-52.

25. Andreyev HJ, Norman AR, Cunningham D, Oates J, Dix BR, lacopetta BJ, et al. Kirsten ras mutations in patients with colorectal cancer: the "RASCAL II" study. Br J Cancer. 2001;85:692-6.

\section{Submit your next manuscript to BioMed Central and take full advantage of:}

- Convenient online submission

- Thorough peer review

- No space constraints or color figure charges

- Immediate publication on acceptance

- Inclusion in PubMed, CAS, Scopus and Google Scholar

- Research which is freely available for redistribution 